\title{
Subjective and objective vestibular changes that occur following paediatric cochlear implantation: systematic review and meta- analysis
}

Michael Yong, Emily Young, Jane Lea, Hannah Foggin, Erica Zaia, Frederick K. Kozak and Brian D. Westerberg ${ }^{*}$

\begin{abstract}
Objective: Cochlear implantation can result in post-operative vestibular dysfunction of unknown clinical significance. The objective of this study was to characterize the presence, magnitude, and clinical significance of vestibular dysfunction that occurs after pediatric cochlear implantation.

Data sources: The databases Embase, Medline (OvidSP), and PubMed were used. Only articles published in English were included. Grey literature and unpublished sources were also reviewed.

Study selection: Articles published from 1980 until the present which documented pre-operative and post-operative vestibular testing on children under the age of 18 were used.

Data extraction: Parameters that were assessed included number of patients, pre- and post-operative vestibularevoked myogenic potentials (VEMPs), head impulse testing (HIT), calorics, and posturography, timing of pre- and postoperative testing, symptomatology, and other demographic data such as etiology of the hearing loss.

Data synthesis: Ten articles were included. Relative risk values evaluating the effect of cochlear implantation on vestibular function were calculated for VEMPs and caloric testing due to the availability of published data. $I^{2}$ values were calculated and 95\% confidence intervals were reported. Separate analyses were conducted for each individual study and a pooled analysis was conducted to yield an overall relative risk. Assessment on risk of bias in individual studies and overall was performed.

Conclusion: Pediatric cochlear implantation is associated with a statistically significant decrease in VEMP responses post-operatively (RR 1.8, $p<0.001, I^{2} 91.86,95 \% C l 1.57-2.02$ ). Similar results are not seen in caloric testing. Insufficient data is available for analysis of HIT and posturography. Further studies are necessary to determine the effect of cochlear implantation on objective vestibular measures post-operatively and whether any changes seen are clinically relevant in this population.
\end{abstract}

\section{Introduction}

Cochlear implantation in the pediatric population has become a routine procedure for children with bilateral severe to profound sensorineural hearing loss [1]. The past decade has seen a rapid increase in the number of implants being performed due to cost-effectiveness studies and the improved quality of life enjoyed by implant recipients $[2,3]$. However, there have been recent efforts aimed at further

* Correspondence: BWesterberg@providencehealth.bc.ca

BC Rotary Hearing and Balance Centre, St. Paul's Hospital, 1081 Burrard St, Vancouver, BC V6Z 1Y6, Canada characterizing the potential side effects of cochlear implantation in children, specifically with respect to resultant vestibular dysfunction. There has been a growing trend towards bilateral simultaneous implantation in children due to recent studies showing an increased ability to preserve normal cortical preference and development, improved sound localization contributing to enhanced preverbal communication and language development, and greater cost-effectiveness $[1,4-6]$. The impact that bilateral implantation has on vestibular and balance function in children has been studied but methodologies and results are 
variable, making it difficult to draw general conclusions from this research.

The vestibular ramifications of implantation in the adult population have been documented [7-9]. Cochlear implantation has the potential to cause a decrease in vestibular function in adults measured by objective testing such as vestibular evoked myogenic potentials (VEMPs), calorics, and head impulse testing (HIT) $[8,9]$. In addition, a recent meta-analysis of vestibular function after cochlear implant surgery reported a significant negative effect on caloric and VEMP testing in adults, although the overall patient-reported symptomatic manifestations of these objective findings were found to be insignificant [10].

Documentation of pre- and post-operative vestibular function surrounding pediatric cochlear implantation are limited and perhaps reflective of the fact that many centres do not routinely perform such testing. This may be due to several reasons such as cost, unclear benefit, difficulty in testing very young children, and lack of available equipment. As such, no prior systematic reviews or metaanalyses are available. While pediatric cochlear implantation has proven to be a relatively safe procedure with few severe long-term complications, those related to the vestibular system have been reported [11, 12]. Data from the literature to date has shown that vestibular end-organ dysfunction can be a risk factor for implant failure in the pediatric population and that there may be value in identifying children with vestibular impairment after surgery to avoid this complication [13]. In addition, recent research has shown that cochlear implantation has the potential to affect vestibular function through direct current spread from the cochlea to the vestibular system [14].

The now commonplace nature of this procedure, including bilateral implantation, and the potential long-term ramifications of undetected vestibular damage highlights a need for systematic analysis of the current literature in order to develop evidence-informed decisions regarding implantation. In turn, this may further elucidate potential contraindications to surgery or highlight the need for earlier vestibular rehabilitation interventions following implantation in young patients.

\section{Objectives}

The aim of this systematic review was to evaluate the vestibular side-effects of cochlear implantation in the pediatric population through examination of objective and subjective vestibular parameters. The review sought to answer the following questions:

(1) Is there a significant decrease in objective vestibular testing responses in the implanted ear after cochlear implantation?
(2) Are there any changes in subjective vestibular symptoms post-operatively that suggest persistent long-term clinical impairment?

According to the PICOS approach, the population of interest included pediatric patients under the age of 18 undergoing cochlear implantation who have had pre-operative and post-operative vestibular testing. Cochlear implantation represented the intervention, and the comparison or control was the pre-operative vestibular status of the patient prior to surgery. The outcome under investigation was the objective and subjective vestibular status of patients after surgery. The review was not limited to any specific study design criterium.

\section{Methods}

Ethics exemption was obtained from the University of British Columbia Research Ethics Board. The Preferred Reporting Items for Systematic Reviews and Meta-analysis (PRISMA) statement was followed during the review.

\section{Protocol}

Methods of analysis and inclusion/exclusion criteria were specified in advance and documented in a separate protocol.

\section{Study inclusion criteria}

Prospective or retrospective cohort studies reporting both pre- and post-operative vestibular testing of pediatric patients under the age of 18 at the time of cochlear implantation were included. Studies including children who underwent either unilateral or bilateral cochlear implantation (simultaneous or sequential) were included. Studies that included adults as well as children were also included, providing individual relevant data was available for the pediatric patients. Children with additional comorbidities were included, even those with conditions that predispose to vestibular dysfunction, as these form an important cohort of patients currently undergoing cochlear implantation.

\section{Study exclusion criteria}

Studies including only either pre- or post-operative testing were excluded. Non-English language papers were excluded as their exclusion has been shown to have limited impact on review results [15]. Publications prior to 1980 were intentionally excluded.

\section{Types of outcome measures}

1) Primary outcomes measures: pre and post operative vestibular function testing including at least one of: VEMPs, calorics, HIT, dynamic posturography. 
2) Secondary outcome measures: subjective symptoms of vestibular function pre- and post-cochlear implantation.

\section{Information sources}

A structured search was performed of the following three databases between 1980 and August 2017: Medline (Ovid), EmBase (Ovid), and PubMed. The reference list of each eligible study was also examined for extraction of relevant published articles not identified during the original search. A search of the grey literature, including unpublished data and $\mathrm{PhD}$ theses was also performed.

\section{Search strategy}

The keywords, MeSH terms, and phrases searched included the following: "cochlear implant", "pediatric", "child" "VEMP", "dizziness", "posturography", "vertigo" and "vestibular". The search strategy was devised collectively and executed independently by two of the study authors (EY; MY). No study design or data limits were imposed upon the search. The full search strategy can be found in Appendix.

\section{Study selection and data collection process}

The search results were collated on a Microsoft Excel (Microsoft, 2013) spreadsheet. The data collection sheet was piloted on two randomly-selected included studies and refined accordingly. Duplicate articles were eliminated. All abstracts were reviewed independently by authors EY and MY for relevance to the study topic. Full text studies of articles deemed to be relevant were further reviewed. Discrepancies regarding the inclusion or exclusion of studies were resolved through discussion between the two aforementioned authors. Neither of the review authors was blinded to the journal titles or the study authors or institutions.

\section{Data items/outcomes}

The selected articles were searched for vestibular outcomes before and following cochlear implantation. VEMP testing, calorics, HIT, and posturography were the primary parameters. Demographic data on individual participants in each of the selected studies were gathered. These included, whenever available, etiology of hearing loss, imaging status, age at implantation, unilateral or bilateral implantation, method of implantation, simultaneous or sequential implantation, and timing of pre- and post-operative vestibular testing. Documentation of subjective post-operative vestibular symptoms was also extracted when possible. The two aforementioned authors independently extracted data from the selected articles according to these predetermined parameters. Any discrepancies in extracted data were resolved by discussion between the review authors.
Risk of bias in individual studies

A checklist including ten key questions adapted from the Critical Appraisal Skills Programme by the Institute of Health at Oxford University was used for the risk of bias assessment [16]. Studies were categorized into one of three categories; (A) Low risk of bias; (B) Medium risk of bias; (C) High risk of bias. Both authors independently examined each study for risk of bias and assigned a categorization. Any discrepancies were resolved through discussion.

An assessment of the risk of meta-bias was also performed. This involved an independent review by both authors focusing on possible selective reporting within studies and a consideration of publication bias.

\section{Summary measures}

The primary targeted measure calculated from the data was the relative risk of cochlear implantation on vestibular dysfunction. This was performed by comparing each child's objective vestibular function pre-operatively to his or her vestibular function post-operatively. Abnormal test results were determined using each study authors' definition.

\section{Planned methods of analysis}

Separate analyses were performed on each individual study, and then a pooled analysis was performed on all studies to yield an overall relative risk. This involved a calculation of relative risk values, as well as $\mathrm{I}^{2}$ calculations and 95\% confidence intervals. Statistical significance was defined as $p<0.05$.

\section{Strength of evidence}

The strength of evidence was determined by the significance of the meta-analysis, relevance of objective testing in the context of clinical impact, and the risk of bias assessment performed on all studies.

\section{Results}

Study selection

Applying the predefined criteria, 410 studies were identified based on initial search of the databases (Fig. 1). An additional three studies were identified based on a review of study references and the grey literature. After duplicates were removed, a review of 408 abstracts resulted in exclusion of 374 studies that did not meet one or more of the inclusion criteria. Of the remaining 34 studies that underwent full text review, 23 were excluded based on participants over the age of 18 or not reporting both preoperative and post-operative vestibular testing. The remaining 11 studies were included for full analysis.

\section{Study characteristics}

Demographic data were extracted from all 11 studies which included study design, number of patients (study size), mean age, timing of pre-operative and post-operative 


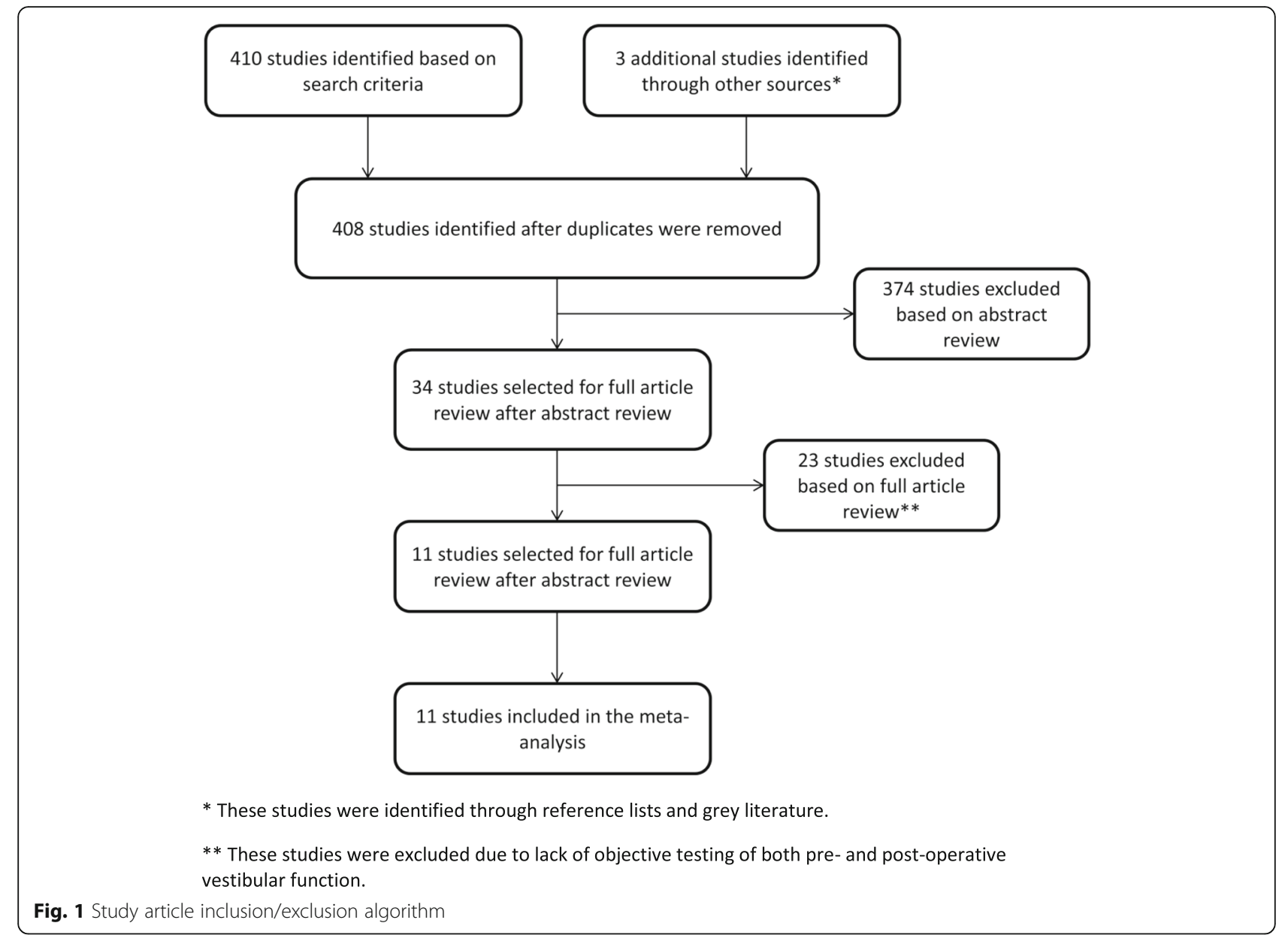

vestibular testing, unilateral or bilateral implantation, sequential or simultaneous implantation, etiology of hearing loss, relevant imaging findings, cochleostomy site, and implant brand/model (Table 1) [18-28].

Data pertaining to pre- and post-operative VEMPs, calorics, HIT, posturography, and subjective symptoms were collected (Table 2). Of the 11 studies, four reported subjective dizziness data and only one reported HIT data. No studies reported posturography data.

\section{Risk of bias within studies}

A formal assessment of the risk of bias was performed on each study (Table 3). After review, 5 studies were considered to have a low risk of bias, 4 had a medium risk of bias, and 2 had a high risk of bias. All studies that were examined were either prospective or retrospective cohort studies.

\section{Results of individual studies}

Data extracted from individual studies were analyzed and relative risk values were calculated for each study. The results for the VEMP analyses are displayed in the Forest plot in Fig. 2. Five of the nine studies [18, 19, 21, 24,
28] that measured cervical VEMP (cVEMP) responses showed a statistically significant increase in abnormal VEMP responses following cochlear implantation. The pooled relative risk of 1.800 was supported by a $95 \%$ confidence interval that did not span 1 (95\%CI 1.442-2.281) and a significant $p$ value of less than 0.001 . On review of all contributing studies, a VEMP change was defined as any significant change in threshold, amplitude, or latency measured post-operatively when that ear had baseline normal VEMP testing. A change was also defined as a complete loss of VEMP responses in an ear that had baseline abnormal VEMP testing (threshold, amplitude, or latency). These results represented Level 3 evidence according to OCEBM [17].

The results for the calorics analysis are displayed in the Forest plot in Fig. 3. One of the three studies [27] that measured calorics showed a statistically significant reduction in caloric responses following implantation. However, the pooled relative risk of 1.012 indicated no overall significant change and was based on only three different studies with the 95\% confidence interval limits widely spanning below and above 1 (95\%CI $0.712-$ 1.439). Additionally, each of the three studies had a 


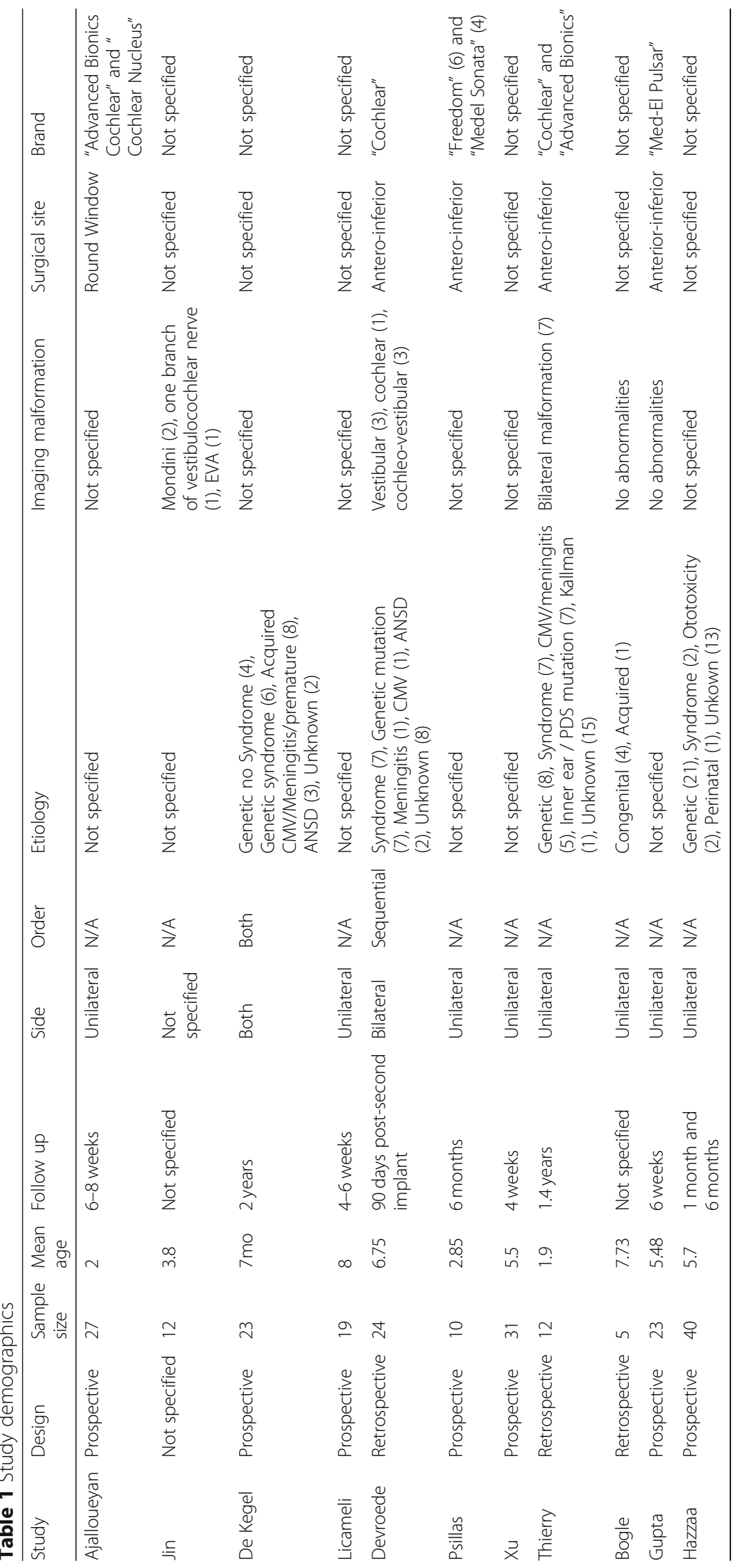


Table 2 Study vestibular findings

\begin{tabular}{|c|c|c|c|c|c|}
\hline Study & VEMP & Calorics & vHIT & Non-video HIT & Symptoms \\
\hline \multirow[t]{2}{*}{ Ajalloueyan } & $\begin{array}{l}\text { Pre: 7\% (2) no CVEMP bilaterally. } \\
\text { 19\% (5) no cVEMP unilaterally. }\end{array}$ & $\begin{array}{l}\text { Pre: } 8 \%(1) \\
\text { dysfunction. }\end{array}$ & \multirow[t]{2}{*}{ Not performed } & Pre: $0 \%$ had dysfunction. & \multirow[t]{2}{*}{ N/A } \\
\hline & Post: $20 \%$ (8) abnormal cVEMP. & Post: No change. & & Post: No change. & \\
\hline \multirow[t]{2}{*}{ Jin } & $\begin{array}{l}\text { Pre: } 8 \% \text { (1) reduced cVEMP, } \\
42 \% \text { (5) no cVEMP. }\end{array}$ & \multirow[t]{2}{*}{ Not performed } & \multirow[t]{2}{*}{ Not performed } & \multirow[t]{2}{*}{ Not performed } & \multirow[t]{2}{*}{ N/A } \\
\hline & $\begin{array}{l}\text { Post: } 100 \% \text { (12) abnormal } \\
\text { cVEMP. }\end{array}$ & & & & \\
\hline \multirow[t]{2}{*}{ De Kegel } & $\begin{array}{l}\text { Pre: } 26 \%(5) \text { had abnormal } \\
\text { cVEMP unilaterally or bilaterally. }\end{array}$ & \multirow[t]{2}{*}{ Not performed } & \multirow[t]{2}{*}{ Not performed } & \multirow[t]{2}{*}{ Not performed } & \multirow{2}{*}{$\begin{array}{l}\text { Significant drop in gross motor } \\
\text { performance, recovering toward } \\
\text { age } 2 \text {. }\end{array}$} \\
\hline & Post: No change. & & & & \\
\hline \multirow[t]{2}{*}{ Licameli } & Pre: $12 \%$ (2) no cVEMP. & \multirow[t]{2}{*}{ Not performed } & \multirow[t]{2}{*}{ Not performed } & \multirow[t]{2}{*}{ Not performed } & \multirow{2}{*}{$\begin{array}{l}\text { Data not reliable collected, but } \\
\text { no significant difference found. }\end{array}$} \\
\hline & Post: $84 \%$ (16) abnormal cVEMP. & & & & \\
\hline \multirow[t]{2}{*}{ Devroede } & Pre: $21 \%$ (5) abnormal cVEMP. & $\begin{array}{l}\text { Pre: } 71 \%(17) \\
\text { abnormal calorics. }\end{array}$ & \multirow[t]{2}{*}{ Not performed } & \multirow[t]{2}{*}{ Not performed } & \multirow{2}{*}{$\begin{array}{l}\text { Post-op dizziness in } 33 \% \text { (3) who } \\
\text { had post-op vestiublar dysfunction, } \\
\text { which subsequently recovered. }\end{array}$} \\
\hline & Post: 38\% (9) abnormal cVEMP. & $\begin{array}{l}\text { Post: } 67 \% \text { (16) } \\
\text { abnormal calorics. }\end{array}$ & & & \\
\hline \multirow[t]{2}{*}{ Psillas } & $\begin{array}{l}\text { Pre: } 30 \% \text { (3) no cVEMP, 30\% } \\
\text { (3) reduced cVEMP. }\end{array}$ & \multirow[t]{2}{*}{ Not performed } & \multirow[t]{2}{*}{ Not performed } & \multirow[t]{2}{*}{ Not performed } & \multirow[t]{2}{*}{$\begin{array}{l}\text { No dizziness, vertigo, instability, } \\
\text { or nystagmus noted post-op. }\end{array}$} \\
\hline & Post: $100 \%$ (10) abnormal VEMP. & & & & \\
\hline \multirow[t]{2}{*}{$\mathrm{Xu}$} & Pre: $26 \%$ (6) abnormal cVEMP. & \multirow[t]{2}{*}{ Not performed } & \multirow[t]{2}{*}{ Not performed } & \multirow[t]{2}{*}{ Not performed } & \multirow[t]{2}{*}{ N/A } \\
\hline & Post: 65\% (15) abnormal VEMP. & & & & \\
\hline \multirow[t]{2}{*}{ Bogle } & Pre: 0\%abnormal cVEMP. & \multirow[t]{2}{*}{ Not performed } & \multirow[t]{2}{*}{ Not performed } & \multirow[t]{2}{*}{ Not performed } & N/A \\
\hline & Post: 20\% (1) abnormal cVEMP. & & & & \\
\hline Gupta & Not performed & $\begin{array}{l}\text { Pre: } 22 \%(5) \text { abnormal } \\
\text { calorics. }\end{array}$ & Not performed & Not performed & N/A \\
\hline & & $\begin{array}{l}\text { Post: } 35 \% \text { (8) abnormal } \\
\text { calorics. }\end{array}$ & & & \\
\hline Hazzaa & $\begin{array}{l}\text { Pre: } 55 \% \text { (22) abnormal or } \\
\text { absent cVEMP. }\end{array}$ & Not performed & Not performed & Not performed & N/A \\
\hline & $\begin{array}{l}\text { Post: } 80 \% \text { (32) abnormal or } \\
\text { absent CVEMP at } 6 \text { months. }\end{array}$ & & & & \\
\hline
\end{tabular}

different definition of what constituted a significant caloric response change. Ajalloueyan et al. did not report a cut-off value and performed calorics using cold air, Gupta et al. defined a difference of $>15 \%$ in monothermal testing as significant and performed calorics using warm air, and Devroede et al. defined a difference of $>20 \%$ in bithermal testing and $>27 \%$ for monthermal testing as significant and performed monothermal calorics using cold water. This was deemed to represent Level 3 evidence.

Only four studies $[18,19,23,30]$ reported on subjective symptoms, and there were no studies that reported outcomes in a quantitative manner. Based on the qualitative reporting of data, no studies indicated that there were any post-operative symptoms such as imbalance or dizziness either immediately or long-term post-operatively.

\section{Synthesis of results}

Data were combined for the purpose of a pooled meta-analysis. Relative risk, $\mathrm{I}^{2}$ value, and the $95 \%$ confidence interval were yielded and displayed together in the Forest plot (Fig. 2). The results showed a statistically significant increase in abnormal VEMP responses after either unilateral or bilateral cochlear implantation (RR 1.8, $p<0.001,95 \%$ CI 1.57-2.02). The heterogeneity in the studies included for analysis was significant $\left(\mathrm{I}^{2}\right.$ 91.86, $\left.\mathrm{Q}=98.304\right)$. A statistically significant increase in abnormal caloric testing was not similarly seen $(p=0.60)$.

\section{Risk of bias across studies}

The risk of bias when comparing between studies was deemed to be low. There were no concerns identified 


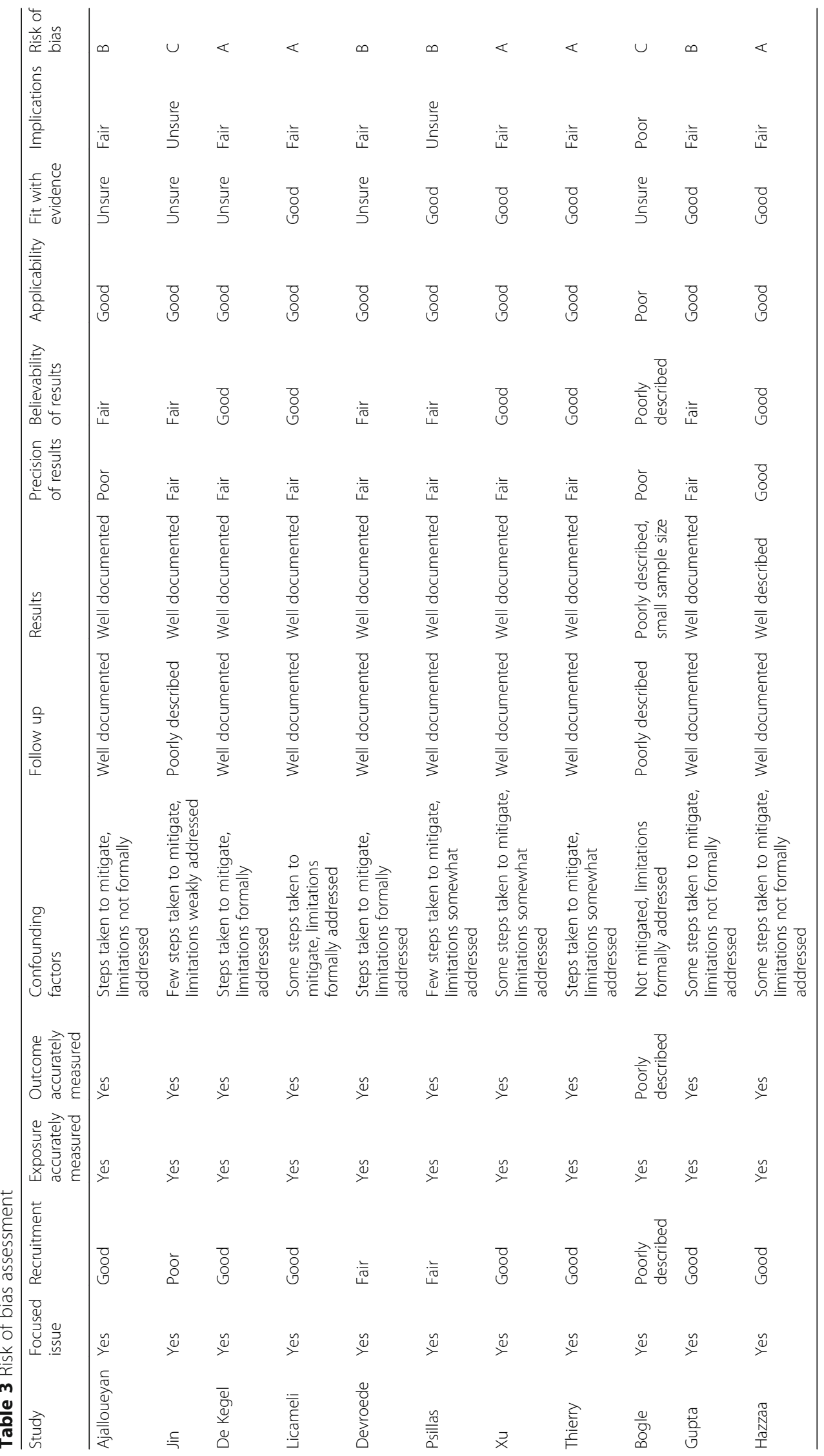




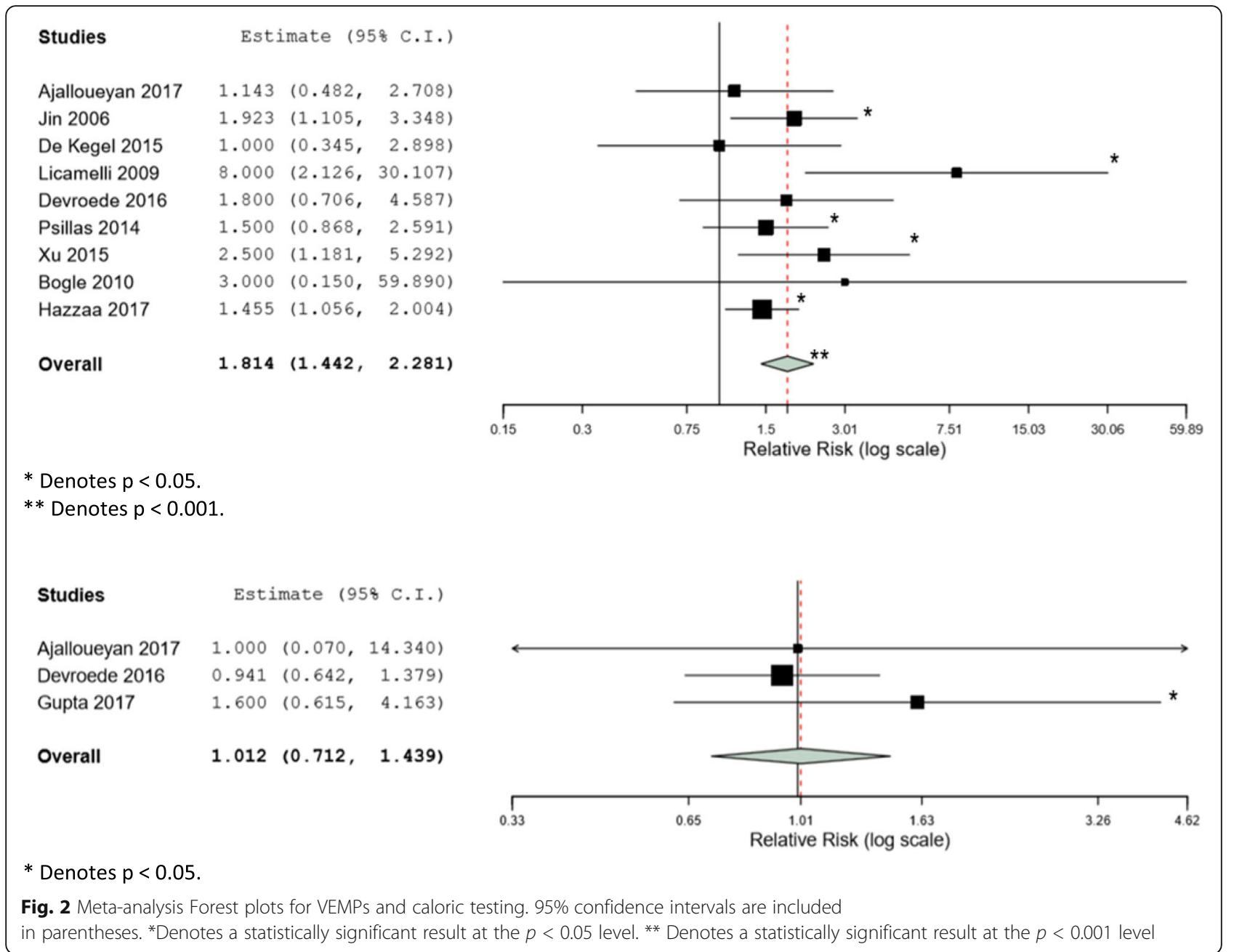

over selective reporting of data, as patients in the studies that were reviewed were generally all accounted for in the results.

\section{Discussion}

\section{Summary of evidence}

Cochlear implantation carries a risk of post-operative vestibular dysfunction. Although its clinical significance currently appears to be minimal, long-term findings in the timeframe of decades have yet to be reported. Data from adult studies suggests that following implant surgery, otolithic organs and canal function can be compromised, leading to potential clinical manifestations such as post-operative imbalance, vertigo, and falls $[1,4,7]$. This is especially concerning for children who are more commonly undergoing bilateral implantation, raising the theoretical concern of bilateral vestibular dysfunction in the long term following surgical intervention. However, recent work on perceptual visual tilt using the static subjective visual vertical test has also shown that electrical pulses from the implant itself after initial cochlear implantation may have a role in actually improving vestibular function by helping to correct abnormal perception of vertical [29].

Cochlear implantation can potentially lead to disruption of saccular function in pediatric patients. Review of the literature would suggest there is a significant risk for increase in abnormal VEMP responses in pediatric patients undergoing cochlear implantation, with a relative risk of $1.8(p<0.001)$ despite several individual studies that did not display significant results $[19,20,22,23,26]$. These findings support similar findings published in a recent systematic review studying vestibular outcomes after adult cochlear implantation, showing a log relative risk of $0.5099(p<0.0001)$ after an analysis of 12 separate studies published since 2008 [9]. Proposed mechanisms for this finding have been previously discussed in the literature and include direct trauma related to insertion of the electrode, intraoperative perilymphatic fluid leakage, electrical stimulation of the otolithic organs, and foreign body labyrinthitis $[17,18]$.

There was no significant change in caloric responses in pediatric patients undergoing cochlear implantation. However, this is based on a small number of available studies 
reporting on this parameter and poor precision of available data. In addition, the caloric testing method and definition of canal dysfunction was widely variable, contributing to a significant source of bias.

There was a lack of available data concerning a number of the parameters of interest, specifically rotary chair, HIT and posturography. Challenges associated with collecting pediatric video-HIT data in particular have been reported in the past, citing difficulties such as lack of interest, involuntary or voluntary tensing of cervical muscles during testing, and problems with keeping eyes open during testing, especially in children under six years of age [30]. A significant proportion of pediatric implantations in this review occurred during the first five to six years of life.

Regardless of the objective results, there was no evidence for subjective symptoms of dizziness occurring in the pediatric population after cochlear implantation. Whether this is a limitation in available data or in the subjective reporting of symptoms, or reflective of a lack of clinically significant injury to the vestibular system in this population is uncertain.

\section{Limitations}

There is a paucity of data on vestibular function in the pediatric population undergoing cochlear implantation; several studies were excluded because of a lack of pre-operative and post-operative objective measurements of vestibular function. As a result, there may be insufficient power to detect statistically significant differences in objective vestibular measures other than VEMPs. In addition, there was a general lack of data on motor function. Posturography can be difficult to perform on children and cannot be performed on young children and infants, which explains the lack of data in this category. Future studies examining vestibular function in implanted children may benefit from examining patients with scales such as the Ghent modification of the Alberta infant motor scale in order to provide age-standardized measures of motor function [30].

The amount of variance or heterogeneity observed among studies analyzing VEMP responses was substantial $\left(\mathrm{I}^{2} 91.86\right.$, $\mathrm{Q}=98.304)$. Reasons could include inherent differences in the testing methods employed by different studies used to determine whether children displayed objective vestibular findings. Some studies reported detailed protocols of what constituted an abnormal VEMP response, including abnormalities in latency, amplitude, or threshold [20, 23, 24], whereas one study simply classified abnormal VEMP responses as hyporeflexic or areflexic [19]. Additionally, the mean time to follow-up was widely variable and ranged from as short as 4 weeks post-implant surgery to as long as two years post-operatively; vestibular dysfunction likely has the ability to recover or compensate over time.

Six of the eleven studies that were included in this review were graded as having a medium or high risk of bias due to poor documentation of follow-up, insufficient measures taken to reduce confounding factors in sample populations, and poor to fair precision of results.

The lack of reporting etiology of hearing loss and imaging findings in many of the studies may be relevant; certain conditions such as an enlarged vestibular aqueduct and other anomalous cochleovestibular anatomy have been shown to be responsible for higher rates of intraoperative CSF leaks, which could potentially put the patient at a higher risk for post-operative vestibular dysfunction [31].

We did not feel that there were significant limitations concerning reporting bias or incomplete retrieval of published research. The number of studies that were included in this analysis approximated the expected number based on similar studies on vestibular outcomes after cochlear implantation in adults [10].

Finally, cochlear implant electrode technology continues to improve and smaller diameter electrodes are facilitating atraumatic cochlear implantation. What role this may have in reducing the potential for vestibular injury in the future is uncertain.

\section{Conclusions}

Pediatric patients experience a statistically significant change in vestibular function post-implantation as measured by VEMPs. This important finding supports previous work showing vestibular changes post-implantation in the adult population. However, data on post-operative subjective symptoms in the pediatric population is currently lacking. Additional longitudinal studies examining the correlation between VEMP changes and clinical symptoms may be warranted to determine the clinical significance of the findings in this review.

\section{Appendix A}

\section{Full search strategy}

The search on PubMed included:

((((cochlear implant [Title/Abstract]) OR cochlear implantation [Title/Abstract])) AND ((((paediatric [Title/Abstract]) OR pediatric [Title/Abstract]) OR child [Title/Abstract]) OR children [Title/Abstract] )) AND $((((()((($ vestibular [Title/Abstract]) OR VEMPs [Title/Abstract]) OR posturography [Title/Abstract]) OR dizziness handicap inventory [Title/Abstract]) OR DHI [Title/Abstract]) OR vertigo [Title/Abstract]) OR balance [Title/Abstract]) OR caloric [Title/ Abstract]) OR vestibule [Title/Abstract]) OR labyrinth [Title/ Abstract])

The search on Medline (Ovid) and Embase (Ovid) included:

((cochlear implant.mp. or exp. Cochlear Implants/) and (pediatric.mp. or exp. Pediatrics/) and (exp Dizziness/ or exp. Vestibule, Labyrinth/ or exp. Vertigo/ or exp. Vestibular Diseases/ or vestibular.mp.) and (exp Child/ or child.mp.)) 


\section{Acknowledgements}

Not applicable.

\section{Funding}

No funding was provided for this study.

\section{Sources of support and disclosure}

Nothing to disclose.

\section{Authors' contributions}

MY drafted the protocol, refined the search algorithm, screened papers, conducted statistical analyses, and drafted the manuscript. EY assisted in drafting the protocol, screening papers, conducting statistical analyses, and revising the manuscript. $J$ formulated the research question and revised the manuscript. HF conducted statistical analyses. EZ revised the manuscript. FKK revised the manuscript. BDW formulated the research question and revised the manuscript. All authors read and approved the final manuscript.

\section{Ethics approval and consent to participate}

Ethics exemption was obtained from the University of British Columbia Research Ethics Board. The Preferred Reporting Items for Systematic Reviews and Meta-analysis (PRISMA) statement was followed during the review.

\section{Consent for publication}

Not applicable.

\section{Competing interests}

The authors declare that they have no competing interests.

\section{Publisher's Note}

Springer Nature remains neutral with regard to jurisdictional claims in published maps and institutional affiliations.

Received: 1 January 2019 Accepted: 23 April 2019

Published online: 22 May 2019

\section{References}

1. Lammers MJW, van der Heijden GJMG, Pourier VEC, Grolman W. Bilateral cochlear implantation in children: a systematic review and best-evidence synthesis. Laryngoscope. 2014;124(7):1694-9.

2. Chen JM, Amoodi H, Mittmann N. Cost-utility analysis of bilateral cochlear implantation in adults: a health economic assessment from the perspective of a publicly funded program. Laryngoscope. 2014;124(6):1452-8.

3. McKinnon BJ. Cost effectiveness of cochlear implants. Curr Opin Otolaryngol Head Neck Surg. 2014;22(5):344-8.

4. Perez-Martin J, Artaso MA, Diez FJ. Cost-effectiveness of pediatric bilateral cochlear implantation in Spain. Laryngoscope. 2017;127(12):2866-72.

5. Gordon KA, Wong DD, Papsin BC. Bilateral input protects the cortex from unilaterally-driven reorganization in children who are deaf. Brain. 2013;136: 1609-25.

6. Papsin BC, Gordon KA. Cochlear implants for children with severe-toprofound hearing loss. N Engl J Med. 2007;357:2380-7.

7. Buchman CA, Joy J, Hodges A, Telischi FF, Balkany TJ. Vestibular effects of cochlear implantation on the vestibular function. Laryngoscope. 2004;114(10 Pt 2 Suppl 103):1-22

8. Karsiari E, Balatsouras DG, Sengas J, Riga M, Korres GS, Xenelis J. Influence of cochlear implantation on the vestibular function. Eur Arch Otorhinolaryngol. 2013;270(2):489-95.

9. Melvin TA, Della Santina CC, Carey JP, Migliaccio AA. The effects of cochlear implantation on vestibular function. Otol Neurotol. 2009;30(1):87-94.

10. Ibrahim I, da Silva SD, Segal B, Zeitouni A. Effect of cochlear implant surgery on vestibular function: meta-analysis study. J Otolaryngol Head Neck Surg. 2017:46(1):44

11. Sivam SK, Syms CA 3rd, King SM, Perry BP. Consideration for routine outpatient pediatric cochlear implantation: a retrospective chart review of immediate post-operative complications. Int J Pediatr Otorhinolaryngol. 2017;94:95-9.

12. Terry B, Kelt RE, Jeyakumar A. Delayed complications after Cochlear implantation. JAMA Otolaryngol Head Neck Surg. 2015;141(11):1012-7.

13. Wolter NE, Gordon KA, Papsin BC, Cushing SL. Vestibular and balance impairment contributes to Cochlear implant failure in children. Otol Neurotol. 2015;36(6):1029-34.
14. Parkes WJ, Gnanasegaram JJ, Cushing SL, McKnight CL, Papsin BC, Gordon KA. Vestibular evoked myogenic potential testing as an objective measure of vestibular stimulation with cochlear implants. Laryngoscope. 2017;127(2):E75-81.

15. Moher D, Pham B, Klassen TP, Schulz KF, Berlin JA, Jadad AR, et al. What contributions do languages other than English make on the results of meta-analyses? J Clin Epidemiol. 2000;53(9):964-72.

16. Critical Appraisal Skills Programme (2017). CASP Checklist. [online] Available at: http://www.casp-uk.net/casp-tools-checklists. Accessed 1 Jan 2018.

17. OCEBM 2011 Levels of Evidence. The Oxford 2011 Levels of Evidence. 2011. http://www.cebm.net/index.aspx?o=5653. Accessed 31 Jan 2018.

18. Licameli G, Zhou G, Kenna MA. Disturbance of vestibular function Attribuatable to Cochlear implantation in children. Laryngoscope. 2009;119:740-5.

19. Devroede B, Pauwels I, Le Bon SD, Monstrey J, Mansbach AL. Interest of vestibular evaluation in sequentially implanted children: preliminary results. Eur Ann Otorhinolaryngol Head Neck Dis. 2016;133S:S7-S11.

20. Ajalloueyan M, Saeedi M, Sadeghi M, Abdollahi FZ. Int J Ped Otorhinolaryngol. 2017;94:100-3.

21. Jin Y, Nakamura M, Shinjo Y, Kaga K. Vestibular-evoked myogenic potentials in cochlear implant children. Acta Otolaryngol. 2006;126(2):164-9.

22. De Kegel A, Maes L, Van Waelvelde H, Dhooge I. Examining the impact of Cochlear implantation on the early gross motor development of children with a hearing loss. Ear Hear. 2014;36(3):113-21.

23. Psillas G, Pavlidou A, Lefkidis N, Vital I, Markou K, Triaridis S, Tsalighopoulos M. Vestibular evoked myogenic potentials in children after cochlear implantation. Auris Nasus Larynx. 2014:41:432-5.

24. Xu X, Zhang X, Zhang Q, Hu J, Chen Y, Xu M. Ocular and cervical vestibularevoked myogenic potentials in children with cochlear implant. Clin Neurophysiol. 2015;126:1624-31.

25. Thierry B, Blanchard M, Leboulanger N, Parodi M, Wiener-Vacher SR, Garabedian E, Loundon N. Cochlear implantation and vestibular function in children. Int J Ped Otorhinolaryngol. 2015;79:101-4.

26. Bogle, J. The effect of Cochlear implantation on the vestibular evoked myogenic potential response in children and adults. Speech, Language, and Hearing Services Graduate Theses and Dissertations. 2010. Paper 4.

27. Gupta A, Raj P. Compensated vestibular dysfunction post Cochlear implantation in children with sensorineural hearing loss: a prospective study. Indian J Otolaryngol Head Neck Surg. 2017. https://doi.org/10.1007/ s12070-017-1054-0.

28. Hazzaa NM, Maksoud AIA, Khalil LH, Wahba HA, Galal IM. Effects of Cochlear implantation on vestibular pathway: follow up study in Cochlear implant children. Glob J Otolaryngol. 2017;8(4):555743. https://doi.org/10.19080/GJO. 2017.08.555743.

29. Gnanasegaram JJ, Parkes WJ, Cushing SL, McKnight CL, Papsin BC, Gordon KA. Stimulation from Cochlear implant electrodes assists with recovery from asymmetric perceptual tilt: evidence from the subjective visual vertical test. Front Integr Neurosci. 2016;10:32.

30. De Kegel A, Baetens T, Peersman W, Maes L, Dhooge I, Waelvelde H. Ghent developmental balance test: a new tool to evaluate balance performance in toddlers and preschool children. Phys Ther. 2012;92:841-52.

31. Hulse R, Hormann K, Servais JJ, Hulse M, Wenzel A. Clinical experience with video head impulse test in children. Int J Pediatr Otoaryinolaryngol. 2015; 79(8):1288-93.

Ready to submit your research? Choose BMC and benefit from:

- fast, convenient online submission

- thorough peer review by experienced researchers in your field

- rapid publication on acceptance

- support for research data, including large and complex data types

- gold Open Access which fosters wider collaboration and increased citations

- maximum visibility for your research: over $100 \mathrm{M}$ website views per year

At $\mathrm{BMC}$, research is always in progress.

Learn more biomedcentral.com/submissions 